\title{
THE QUOTIENT SEMILATTICE OF THE RECURSIVELY ENUMERABLE DEGREES MODULO THE CAPPABLE DEGREES
}

BY

\author{
STEVEN SCHWARZ
}

\begin{abstract}
In this paper, we investigate the quotient semilattice $R / M$ of the r.e. degrees modulo the cappable degrees. We first prove the $R / M$ counterpart of the Friedberg-Muchnik theorem. We then show that minimal elements and minimal pairs are not present in $R / \underline{M}$. We end with a proof of the $\underline{R} / \underline{M}$ counterpart to Sack's splitting theorem.
\end{abstract}

0. Introduction. The set of all r.e. degrees is made into an upper semilattice (with 0 and 1) in a natural way: namely, the reducibility relation between r.e. sets induces a partial ordering on degrees, for which it is readily shown that finite suprema always exist. This semilattice structure, denoted " $R$ ", has been extensively studied.

Earliest results stress the richness and the uniformity of $\underline{R}$. For instance, the Friedberg-Muchnik theorem states that there exists an incomparable pair in $\underline{R}$ (Friedberg [1957] and Muchnik [1956]). This may be extended to obtain the existence of a countably infinite independent set in $\underline{R}$. It then follows that any countable partial ordering may be embedded into $\underline{R}$ (Sacks [1966]). Another example: Sacks' splitting theorem states that any element of $\underline{R}$ may be written as the supremum of an imcomparable pair. In fact, such a pair may always be chosen to lie outside any preassigned (nontrivial) principal filter (Sacks [1963]). A corollary of this is that every element of $\underline{R}$ (except the least and greatest elements) is half of an incomparable pair.

The culmination of the early uniformity results about $\underline{R}$ is Sacks' density theorem, which states that, whenever $\underline{a}$ and $\underline{b}$ are elements of $\underline{R}$ such that $\underline{a}<\underline{b}$, then $\underline{c}$ can be found in $\underline{R}$ such that $\underline{a}<\underline{c}<\underline{b}$ (Sacks [1964]). J. Shoenfield [1965] responded to this result by conjecturing its strongest possible generalization: namely, that whenever $\underline{a}, \ldots, \underline{a}_{n}$ satisfy a diagram $D=D\left(x_{1}, \ldots, x_{n}\right)$ in $\underline{R}$ and $D_{1}=D_{1}\left(x_{1}, \ldots, x_{n}, y\right)$ is a diagram which xtends $D$, and which is suitably consistent (i.e. it actually occurs in some upper semilattice with 0 and 1 ), then $\underline{b}$ can be found in $\underline{R}$ such that $\underline{a}_{1}, \ldots, \underline{a}_{n}$, $\underline{b}$ satisfy $D_{1}$ in $\underline{R}$.

Shoenfield's conjecture was refuted (independently) by Lachlan [1966] and C. E. M. Yates [1966], who exhibited a minimal pair in $\underline{R}-$ i.e. a pair of elements whose infimum happens to exist and equals the least element. From this point onward, most of the results about $\underline{R}$ stress the pathology of its structure.

Received by the editors June 3, 1982.

1980 Mathematics Subject Classification. Primary 03D25, 03D30.

(C)1984 American Mathematical Society $0025-5726 / 84 \$ 1.00+\$ .25$ per page 
One notable recent exception to pathology results about $\underline{R}$ is an algebraic decomposition of $\underline{R}$ as the disjoint union of two sets of degrees $-\underline{M}$, the set of r.e. degrees which are halves of minimal pairs, and $\underline{P}$, the set of r.e. degrees which contain a promptly simple set-the former being an ideal, the latter a filter in $R$. This decomposition was pieced together by several researchers and was quite unexpected. W. Maass [1982] has originally defined the notion of a promptly simple set as a computational complexity-theoretic version of the simple set notion of $\mathrm{E}$. Post. Maass had used promptly simple sets for his work on the automorphisms of the lattice of all r.e. sets (not degrees). Maass, Shore and M. Stob [1981] later investigated the degrees which contain promptly simple sets and found that they form a filter in $\underline{R}$. The final link was provided by K. Ambos-Spies, Jockusch, Shore, and Soare [to appear], who showed that the degrees containing promptly simple sets and certain other, previously studied collections of degrees all coincide, and that the complement of this common class, $\underline{M}=\underline{R}-\underline{P}$, forms an ideal in $\underline{R}$.

When this algebraic decomposition produced the first interesting ideal in $\underline{R}$, we adopted the suggestion of Jockusch, Shore and Soare, and began the investigation of the quotient semilattice $\underline{R} / \underline{M}$. We have obtained $\underline{R} / \underline{M}$ counterparts to the Friedburg-Muchnik theorem, to Sacks' splitting theorem, and to the usual extensions and corollaries of these results. Thus $\underline{R} / \underline{M}$ contains within it a copy of every countable partial ordering, and every element of $\underline{R} / \underline{M}$ (except the least and greatest) is half of an incomparable pair. We leave open the question of whether the counterpart to Sacks' density theorem holds; the next step in the investigation of $\underline{R} / \underline{M}$ should be to settle this question.

We note that the minimal pair phenomenon is not present in $\underline{R} / \underline{M}$. Thus, dividing once by (halves of) minimal pairs gets rid of them entirely. This leads to the hope that the $\underline{R} / \underline{M}$ counterpart to Shoenfield's conjecture holds, though we leave this as an open question.

One motivation for looking at $\underline{R} / \underline{M}$ is the hope of finding a natural, degree-theoretic semilattice satisfying Shoenfield's conjecture. It may be remarked that, if the search for such a semilattice were one's only goal, one might try substracting $M$ from $\underline{R}$ rather than dividing $\underline{R}$ by $\underline{M}$. However, Ambos-Spies [1980] showed that $\underline{P}=\underline{R}$ $-\underline{M}$ does not satisfy Shoenfield's conjecture, because of the presence of branching degrees in $\underline{P}$. (A degree is branching if it may be written as the infimum of an incomparable pair; for example, the existence of minimal pairs means that the least degree in $\underline{R}$ is branching.)

This paper is devoted to a presentation of our results on $\underline{R} / \underline{M}$. In $\S 1$, we catalog the basic definitions and facts about prompt simplicity which are needed in the study of $\underline{R} / \underline{M}$. Then in $\S 2$, we prove the $\underline{R} / \underline{M}$ counterpart to the Friedberg-Muchnik theorem. In $\S 3$, we show that minimal elements and minimal pairs are not present in $\underline{R} / \underline{M}$. Finally, in $\S 4$, we prove the $\underline{R} / \underline{M}$ counterpart to Sacks' splitting theorem.

1. Definitions and preliminaries. In this section we list the basic definitions and facts about prompt simplicity which are prerequisite for the study of $\underline{R} / \underline{M}$. For a more detailed treatment and for proofs of results we cite without proof, see Ambos-Spies, Jockusch, Shore, and Soare [to appear]. 
Definition 1.1. A coinfinite r.e. set $A$ is promptly simple if there is an enumeration $\left\{A_{s}: s \in \omega\right\}$ of $A$ and a total recursive function $p$ such that, for all $e \in \omega$,

$$
W_{e} \text { infinite } \Rightarrow(\exists x, s)\left[x \in W_{e, \text { at } s} \cap A_{p(s)}\right] .
$$

Here $\left\{W_{e, s}: e, s \in \omega\right\}$ is a uniform standard approximation to all r.e. sets, and $W_{e, \text { at } s}=W_{e, s}-W_{e, s-1}$. An r.e. degree is promptly simple if it contains a promptly simple set. Let $\underline{P}$ denote the class of promptly simple degrees.

Definition 1.2. An r.e. degree $\underline{c}$ is cappable if $\underline{c}=\underline{0}$ or $\underline{c}$ is half of a minimal pair, i.e. there exists a degree $\underline{m}$ incomparable with $\underline{c}$ such that the infimum of $\underline{c}$ and $\underline{m}$ exists and equals $\underline{0}$. Let $\underline{M}$ denote the class of cappable degrees.

THEOREM 1.3. The r.e. degrees $\underline{R}$ may be decomposed into the disjoint union of the classes $\underline{M}$ and $\underline{P}$. The class $\underline{M}$ forms an ideal in $\underline{R}$ (i.e. $\underline{M}$ is closed downward and also under join). The class $\underline{P}$ forms a strong filter in $\underline{R}$ (i.e. $\underline{P}$ is closed upward and, given $\underline{a}$ and $\underline{b} \in \underline{P}$, there exists $\underline{c} \in \underline{P}$ with $\underline{c} \leqslant \underline{a}$ and $\underline{c} \leqslant \underline{b}$ ).

Definition 1.4. For every r.e. degree $\underline{a}$ let $\underline{a}_{M}$ denote the set $\{\underline{b} \in \underline{R}$ : $(\exists \underline{m}, \underline{n} \in \underline{M})[\underline{a} \vee \underline{m}=\underline{b} \vee \underline{n}]\}$. Let $\underline{R} / \underline{M}$ denote the set $\left\{\underline{a}_{\underline{M}}: \underline{a} \in \underline{R}\right\} . \underline{R} / \underline{M}$ carries the structure of an upper semilattice with 0 and 1 as follows:

(1) $\underline{a}_{\underline{M}} \leqslant \underline{b}_{\underline{M}} \Leftrightarrow(\exists \underline{m} \in \underline{M})[\underline{a} \leqslant \underline{b} \vee \underline{m}]$,

(2) $\left(\underline{a}_{\underline{M}}\right) \vee\left(\underline{b}_{\underline{M}}\right)=(\underline{a} \vee \underline{b})_{\underline{M}}$,

(3) $0=\underline{0}_{M}$, and

(4) $1=\underline{0}_{M}^{\prime}$.

LEMMA 1.5. If $C$ is promptly simple, then there is some recursive enumeration $\left\{C_{s}: s \in \omega\right\}$ of $C$ such that for all $e \in \omega$,

$$
W_{e} \text { infinite } \Rightarrow\left(\exists^{\infty} x, s\right)\left[x \in W_{e, \text { at } s} \cap C_{s+1}\right] .
$$

The notation " $\left(\exists^{\infty} x, s\right)$ " means " there exist infinitely many pairs $x, s$. ."

LEMMA 1.6 (PROMPTLY SIMPLE DEGREE LEMMA). Suppose given a recursive enumeration $\left\{A_{s}: s \in \omega\right\}$ for an r.e. set $A$ and a recursive function $p$ such that for all $e \in \omega$

$$
W_{e} \text { infinite } \Rightarrow(\exists x, s)\left[x \in W_{e, \text { ats }} \& A_{s}[x] \neq A_{p(x)}[x]\right] .
$$

Then $A$ is of promptly simple degree.

LEMMA 1.7 (SLOW-DOWN LEMMA). Let $\left\{E_{e}: e \in \omega\right\}$ be a recursive array of r.e. sets. Then there is a recursive function $g$ such that, for all $e \in \omega, W_{g(e)}=E_{e}$ and for all $s \in \omega, W_{g(e), s+1}$ is contained in $E_{e, s}$. (The point is that elements appear in $W_{g(e)}$ strictly after they appear in $E_{e}$.) Furthermore, an index for the function $g$ may be computed effectively from an index for the array $\left\{E_{e}: e \in \omega\right\}$.

REMARK 1.8. It is legitimate to assume that the function $g$ of Lemma 1.7 is available for use during the construction of the array $\left\{E_{e}: e \in \omega\right\}$. To see this, first note that, by the recursion theorem, it is legitimate to assume that an index for the array $\left\{E_{e}: e \in \omega\right\}$ is available; then apply the uniformity of the passage from the array $\left\{E_{e}: e \in \omega\right\}$ to the function $g$. 
2. Existence of incomparable pairs in $\underline{R} / \underline{M}$. In this section we prove the $\underline{R} / \underline{M}$ counterpart to the Friedberg-Muchnik theorem. We then observe that the same method of proof will yield a stronger result and we shall derive as a corollary that any countable partial ordering can be embedded into $\underline{R} / \underline{M}$.

THEOREM 2.1. There exists an incomparable pair in $\underline{R} / \underline{M}$.

Proof. We shall constuct r.e. sets $A_{0}$ and $A_{1}$ such that $\left(\operatorname{deg} A_{0}\right)_{\underline{M}}$ and $\left(\operatorname{deg} A_{1}\right)_{\underline{M}}$ are incomparable in $\underline{R} / \underline{M}$. For each $m \in\{0,1\}$ and each $e, i \in \omega$, we shall meet the requirement

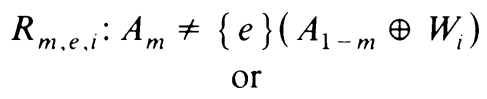

$W_{i}$ is of promptly simple degree.

To see that this suffices, suppose that all requirements $R_{m, e, i}$ are met, but $\left(\operatorname{deg} A_{0}\right)_{\underline{M}}$ $\leqslant\left(\operatorname{deg} A_{1}\right)_{\underline{M}}$, say. Then, for some $\underline{m} \in \underline{M}, \operatorname{deg} A_{0} \leqslant_{T} \operatorname{deg} A_{1} \vee \underline{m}$. Let $i$ be such that $w_{i} \in \underline{m}$; let $e$ be such that $A_{0}=\{e\}\left(\bar{A}_{1} \oplus W_{i}\right)$. Then requirement $R_{0, e, i}$ was not met, a contradiction.

Our primary goal in attacking requirement $R_{0, e, i}$ (of course, $R_{1, e, i}$ is similar) is to obtain a disagreement between $A_{0}$ and $\{e\}\left(A_{1} \oplus W_{i}\right)$. We might try to do this as follows. Look for a witness $x$ such that $\{e\}\left(A_{1} \oplus W_{i} ; x\right)=0$; put $x$ into $A_{0}$; then try to preserve the computation $\{e\}\left(A_{1} \oplus W_{i} ; x\right)$ by means of $A_{1}$-restraint alone. Now $A_{1}$-restraint alone may not suffice to preserve the computation, since the given set $W_{i}$ is free to change at any time. Of course, if $W_{i}$ never changes below $u=$ use $\left(A_{1} \oplus W_{i} ; e, x\right)$, then we shall have a disagreement, and we shall have met the requirment $R_{0, e, i}$. (Here use $\left(A_{1} \oplus W_{i} ; e, x\right)$ is the least number whose membership in the oracle $A_{1} \oplus W_{i}$ is not tested during the computation $\{e\}\left(A_{1} \oplus W_{i} ; x\right)$.) But if $W_{i}$ ever changes below the use $u$, so that $\{e\}\left(A_{1} \oplus W_{i} ; x\right)$ can be corrected to output 1 rather than 0 , then we have to attack all over again with some new witness $x^{\prime}$.

Now we might try to attack as above with an infinite sequence of witnesses $x, x^{\prime}$, $x^{\prime \prime}, \ldots$, and unfortunately fail to get a disagreement with any of them, because every time we try to preserve a computation, a change occurs in $W_{i}$ below the use in that computation. If so, then our primary goal of obtaining a disagreement is hopeless, but we have not necessarily lost all hope of meeting requirement $R_{0, e, i}$. For we can exploit the fact that $W_{i}$ always changes to attain our secondary goal of building a total recursive function $p_{0, e, i}$ such that $W_{i}$ is of promptly simple degree via $p_{0, e, i}$-i.e. such that for all $j$ such that $W_{j}$ is infinite, we have

$$
(*)_{j}:(\exists y, s)\left[y \in W_{j, \text { at } s} \& W_{i, s}[y] \neq w_{i, p_{0 . e .}(s)}[y]\right],
$$

so that, by Lemma 1.6, we may conclude that $W_{i}$ is of promptly simple degree.

Roughly, the idea is as follows. We start an attack at stage $s+1$ with a witness $x$ only if we are also ready to attack $(*)_{j}$ for some $j-$ i.e. we have chosen some $y$ and $j$, such that $y \in W_{j . a t s}$ and $y \geqslant u=u$ use $\left(A_{1} \oplus W_{i} ; e, x\right)$. An attack begun at stage $s+1$ will still end as before at the first stage $t+1 \geqslant s+1$ when $W_{i, t+1}[u] \neq W_{i, s}[u]$. But at that point, $W_{i, t+1}[y] \neq W_{i, s}[y]$ as well, since $y \geqslant u$, so we let $P_{0, e, i}(s)$ be $t+1$, 
winning $(*)_{j}$. Since $W_{i}$ always changes, we will complete infinitely many attacks, winning $(*)_{j}$ for each $j$ such that $W_{j}$ is infinite.

We stress that our interest in the function $p_{0, e, i}$ is entirely contingent upon our failure to achieve any disagreement between $A_{0}$ and $\{e\}\left(A_{1} \oplus W_{i}\right)$. In particular, while we have a disagreement, we do not care whether the function $p_{0, e, i}$ is total or $W_{i}$ is really of promptly simple degree via $p_{0, e, i}$.

Requirement $R_{m, e, i}$ is divided up into infinitely many requirements $S_{m, e, i, j}$, with $j$ varying over $\omega$. Requirement $R_{m, e, i}$ is equivalent to the conjunction of all the requirements $\left\{S_{m, e, i, j}: j \in \omega\right\}$ together with the condition that if

$$
A_{m}=\{e\}\left(A_{1-m} \oplus W_{i}\right),
$$

then the function $p_{m, e, i}$ turns out to be total.

$$
\begin{gathered}
S_{m, e, i, j}: A_{m} \neq\{e\}\left(A_{1-m} \oplus W_{i}\right) \\
\text { or } \\
(\exists y, s)\left[y \in W_{j, \text { at } s} \& W_{i, s}[y] \neq w_{i, p_{m}, e, t}[y]\right] \\
\text { or } \\
W_{j} \text { is finite. }
\end{gathered}
$$

(In other words, if $A_{m}=\{e\}\left(A_{1-m} \oplus W_{i}\right)$ and $W_{j}$ is infinite, then there is an element $y$ and a stage $s$, such that $y$ enters $W_{j}$ at stage $s$ and $W_{i}$ permits $y$ between stage $s$ and stage $p_{m, e, i}(s)$.)

Here is the strategy for a single requirement $S_{0, e, i, j} .\left(S_{1, e, i, j}\right.$ is similar.) We begin by monitoring $\{e\}\left(A_{1} \oplus W_{i} ; x\right)$, for some number $x \notin A_{0}$. If $A_{0}$ and $\{e\}\left(A_{1} \oplus W_{i}\right)$ agree on $x$, then we shall eventually see a convergent computation $\{e\}\left(A_{1} \oplus W_{i} ; x\right)$ $=0$. If so, then we begin waiting for some number $y \geqslant u$, where

$$
u=\operatorname{use}\left(A_{1} \oplus W_{i} ; e, x\right),
$$

to show up in $W_{j}$, say at stage $s$. Then at stage $s+1$ we put $x$ into $A_{0}$ and impose restraint upon $A_{1}$ through the use $u$. If $\{e\}\left(A_{1} \oplus W_{i} ; x\right)$ is ever later corrected to output 1 rather than 0 , it can only be that $W_{i}$ has changed below $u$. If this ever happens, say at stage $t+1 \geqslant s+1$, then we define $p_{0, e, i}(s)$ to be $t+1$.

It is readily seen that this strategy will meet requirement $S_{0, e, i, j}$. Let us denote by $r_{m, e, i, j}$ the restraint imposed on $A_{1-m}$ for the sake of requirement $S_{m, e, i, j}$.

As we try to put all the requirements $S_{m, e, i, j}$ together, we run into mild conflicts. These can be handled with the priority ranking in which $S_{m, e, i, j}$ is of higher priority than $S_{m^{\prime}, e^{\prime}, i^{\prime}, j^{\prime}}\left(\right.$ denoted $\left.S_{m, e, i, j}>S_{m^{\prime}, e^{\prime}, i^{\prime}, j^{\prime}}\right)$ just if $\langle m, e, i, j\rangle\left\langle\left\langle m^{\prime}, e^{\prime}, i^{\prime}, j^{\prime}\right\rangle\right.$. There will be only finitely many injuries to any requirement $S_{m, e, i, j}$.

We say that requirement $S_{m, e, i, j}$ requires attention at stage $s+1$ if the restraint $r_{m, e, i, j}=-1$ and there exist $x=\left\langle x^{\prime}, e, i, j\right\rangle$ and $y \in \omega$ such that all of the following were true at the end of stage $s$ :

(1) $\{e\}\left(A_{1-m} \oplus W_{i} ; x\right)=0$;

(2) $x \notin A_{m}$;

(3) $x \geqslant \max \left\{r_{1-m, e^{\prime}, i^{\prime}, j^{\prime}}: S_{1-m, e^{\prime}, i^{\prime}, j^{\prime}}>S_{m, e, i, j}\right\}$; 
(4) $y \geqslant \operatorname{use}\left(A_{1-m} l \oplus W_{i} ; e, x\right)$;

(5) $y \in W_{\text {j.ats }}$.

Here is the actual construction.

Construction. Stage $s=0$. (Do nothing.) For each $m \in\{0,1\}$ and each $e, i$ and $j \in \omega$, set the restraint $r_{m, e, i, j}$ to -1 .

Stage $s+1$.

Step 1. (Begin at most one attack.) If some requirement $S_{m, e, i, j}$ requires attention at stage $s+1$, then choose the strongest requirement $S_{m, e, i, j}$ which requires attention and fix $x$ and $y$ so that $\langle x, y\rangle$ is as small as possible. Put $x$ into $A_{m}$. Appoint the tuple $\langle y, s, m, e, i, j\rangle$ as an attacker for requirement $S_{m, e, i, j}$. Set the restraint $r_{m, e, i . j}$ to $u=\operatorname{use}\left(A_{1-m} \oplus W_{i} ; e, x\right)$. Cancel all attacks and reset restraints to -1 for all requirements weaker than requirement $S_{m, e, i, j}$.

Step 2. (End attacks.) Now for each requirement $S_{m, e, i, j}$ with an uncancelled attacker $\left\langle y, s^{\prime}, m, e, i, j\right\rangle$, check to see if $W_{i, s+1}[y] \neq W_{i, s}[y]$; if so, then end the attack by defining $p_{m, e, i}\left(s^{\prime}\right)$ to be $s+1$ and cancelling the attacker $\left\langle y, s^{\prime}, m, e, i, j\right\rangle$. To make the function $p_{m, e, i}$ total, also define $p_{m, e . i}$ to be $s+1$ on all arguments less than $s^{\prime}$ for which $p_{m, e, i}$ is not yet defined. (Notice that we do not reset the restraint $r_{m, e, i, j}$ to -1 ; the fact that $r_{m, e, i, j} \geqslant 0$ prevents requirement $S_{m, e, i, j}$ from ever requiring attention again.)

END OF CONSTRUCTION.

The proof is completed by means of a sequence of lemmas.

LEMMA 2.2. Each requirement $S_{m, e, i, j}$ requires attention only finitely often; hence, each restraint $r_{m, e, i, j}$ has a final value $\lim r_{m, e, i, j}$.

Proof. Fix $m, e, i$, and $j$, and inductively assume that $s_{0}$ is a stage so late that no requirement $S_{m^{\prime}, e^{\prime}, i^{\prime}, j^{\prime}}>S_{m, e, i, j}$ ever requires attention after stage $s_{0}$. If requirement $S_{m, e, i, j}$ requires attention at some stage $s+1>s_{0}(s+1$ minimal $)$, then it will receive attention at stage $s+1$ and the restraint $r_{m, e, i, j}$ will assume some value $u \geqslant 0$. Then, by induction on stages $t+1 \geqslant s+1$, requirement $S_{\text {m.e.i.j }}$ will not require attention at stage $t+1$, and the restraint $r_{m, e, i, j}$ will still be $u$ at stage $t+1$.

LEMMA 2.3. Each requirement $S_{m, e, i, j}$ is met.

Proof. We distinguish two cases.

Case $1 . \lim r_{m, e, i, j} \geqslant 0$.

Let $s+1$ be the last stage at which requirement $S_{m, e, i, j}$ receives attention; let $x$ and $y$ be the numbers through which it receives attention. Then there is a disagreement between $A_{m}(x)$ and $\{e\}\left(A_{1-m} \oplus W_{i} ; x\right)$ at the end stage $s+1$ unless $W_{i, s+1}[y] \neq W_{i, s}[y]$. Now, the attacker $\langle y, s, m, e, i, j\rangle$ is never cancelled by injury, so by induction on stages $t+1 \geqslant s+1$, either $A_{m}(x)$ and $\{e\}\left(A_{1-m} \oplus W_{i} ; x\right)$ disagree at the end of stage $t+1$ or else $W_{i, t+1}[y] \neq W_{i, t}[y]$. So in any event, requirement $S_{m, e, i, j}$ is met.

Case 2. $\lim r_{m, e, i, j}=-1$.

Fix a stage $s+1$ so late that the restraint $r_{m, e, i, j}$ and each restraint $r_{m^{\prime}, e^{\prime}, i^{\prime}, j^{\prime}}$ with $S_{m^{\prime}, e^{\prime}, i^{\prime}, j^{\prime}}>S_{m, e, i, j}$ all have reached their final values by the end of stage $s$. Suppose 
for a contradiction that requirement $S_{m, e, i, j}$ is not met. Then there must be a number $x>\max \left\{\lim r_{m^{\prime}, e^{\prime}, i^{\prime}, j^{\prime}}: S_{m^{\prime}, e^{\prime}, i^{\prime}, j^{\prime}}>S_{m, e, i, j}\right\}$ which never enters $A_{m}$. Fix such an $x$. Then there is a first stage $s^{\prime}+1 \geqslant s+1$ such that $\{e\}\left(A_{1-m} \oplus W_{i} ; x\right)$ converges and outputs 0 at stage $s^{\prime}$. Let $u=\operatorname{use}\left(A_{1-m} \oplus W_{i} ; e, x\right)$. $W_{j}$ is finite (since requirement $S_{m, e, i, j}$ is not met) so there is some number $y>u$ at such that $y \in W_{j, \text { at } t}$ for some $t^{\prime} \geqslant s^{\prime}$. But then requirement $S_{m, e, i, j}$ requires and receives attention and the restraint $r_{m, e, i, j}$ takes on a value $u \geqslant 0$ sometime after stage $s^{\prime}+1$, contradicting the choice of the stage $s+1$.

LEMMA 2.4. Each requirement $R_{m, e, i s}$ is met.

Proof. The only thing left to observe is that if $A_{m}=\{e\}\left(A_{1-m} \oplus W_{i}\right)$, then the function $p_{m, e, i}$ turns out to be total. This is clear from Step 2 of the construction.

This concludes the proof of the theorem.

The following is a straightforward extension of the previous theorem.

THEOREM 2.5. There exists an infinite r.e. independent sequence of elements in $\underline{R} / \underline{M}$.

Proof. Using the methods of the proof of Theorem 2.1, we can build an r.e. sequence of r.e. sets $\left\{A_{m}: m \in \omega\right\}$ such that for each $m, e, i \in \omega$, the following requirement is met:

$$
\begin{gathered}
R_{m, e, i}: A_{m} \neq\{e\}\left(\oplus\left\{A_{m^{\prime}}: m^{\prime} \neq m\right\} \oplus W_{i}\right) \\
\text { or } \\
W_{i} \text { is of promptly simple degree. }
\end{gathered}
$$

The details are left to the reader.

Corollary 2.6. Any countable partial ordering may be embedded into $\underline{R} / \underline{M}$.

Proof. Let $P=\left(X, \leqslant_{P}\right)$ be a countable partial ordering; without loss of generality, we may assume that $X=\omega$ and that $\leqslant_{P}$ is recursive. (This follows from two facts: (1) any countable partial ordering may be embedded into the countable atomless Boolean algebra; and (2) there exists a recursively-presented countable atomless Boolean algebra.) Using the r.e. sets $\left\{A_{m}: m \in \omega\right\}$ from the preceding theorem, define a map $f$ from $P$ to $\underline{R} / \underline{M}$, by

$$
f(i)=\left(\underline{a}_{i}\right)_{\underline{M}}, \quad \text { where } \underline{a}_{i}=\operatorname{deg}\left(\oplus\left\{A_{j}: j \leqslant_{P} i\right\}\right) .
$$

The reader may easily verify that $i \leqslant_{P} j$ implies that $\left(\underline{a}_{i}\right)_{\underline{M}} \leqslant\left(\underline{a}_{j}\right)_{\underline{M}}$ and $i{ }_{P} j$ implies that $\left(\underline{a}_{i}\right)_{\underline{M}} \nless\left(\underline{a}_{j}\right)_{\underline{M}}$. (It should be noted that the first of these implications requires that $\leqslant_{P}$ is recursive, as does the conclusion that $\underline{a}_{i}$ is an r.e. degree.) We may take $f$ as the desired embedding.

3. Nonexistence of minimal elements in $\underline{R} / \underline{M}$. This section is devoted to proving that there are no minimal elements in $\underline{R} / \underline{M}$. This result is obtained from an apparently weaker result on avoiding principal filters by applying the strong filter property of $\underline{P}$. This strong filter property of $\underline{P}$ is then also used to remark that 
minimal pairs, minimal triples, etc. are not present in $\underline{R} / \underline{M}$. Our first goal is the following

THEOREM 3.1. (Avoiding principal filters.) Given $\underline{c}_{\underline{M}}>\underline{0}_{\underline{M}}$, there exists $\underline{a}_{\underline{M}}>\underline{0}_{\underline{M}}$ such that $\underline{a}_{\underline{M}} \ngtr \underline{c}_{\underline{M}}$.

Proof. Let $\underline{c}_{\underline{M}}>\underline{0}_{\underline{M}}$ be given. Then $\underline{c}$ is a promptly simple degree, so there exists a promptly simple set $C \in \underline{c}$. Without loss of generality, we may assume given a recursive enumeration of $C$ for which $p(s)=s+1$ is a prompt simplicity function. We shall construct an r.e. set $A$ such that $A$ is also promptly simple via $p(s)=s+1$ and such that $(\operatorname{deg} A)_{\underline{M}} \ngtr \underline{c}_{\underline{M}}$.

To make $A$ promptly simple, we shall meet the following requirement for all $n \in \omega:$

$$
\begin{gathered}
P_{n}:(\exists y, s)\left[y \in \underset{n, \text { ats }}{\text { or }} \& y \in A_{s+1}\right] \\
W_{n} \text { is finite. }
\end{gathered}
$$

(In other words, if $W_{n}$ is infinite, then there exists an element $y$ and a stage $s$, such that $y$ enters $W_{n}$ at stage $s$ and $y$ enters $A$ at stage $s+1$.)

The strategy for a single requirement $P_{n}$ is very simple. If requirement $P_{n}$ has not already been met, then just wait for a large number $y$ to show up in $W_{n}$. If this ever happens, say at stage $s$, then put $y$ into $A$ at stage $s+1$.

Making sure that $(\operatorname{deg} A)_{\underline{M}} \neq \underline{c}_{\underline{M}}$ is more intricate. We must meet, for all $e, i \in \omega$, the requirement

$$
\begin{aligned}
& N_{e, i}: C \neq\{e\}\left(A \oplus W_{i}\right) \\
& \text { or }
\end{aligned}
$$

$W_{i}$ is of promptly simple degree.

Our main goal as we attack requirement $N_{e, i}$ is to obtain a disagreement between $C$ and $\{e\}\left(A \oplus W_{i}\right)$. We might do this by first finding some number $x$ such that for all $z<x,\{e\}\left(A \oplus W_{i} ; z\right)=C(z)$; then putting one of these $z<x$ into $C$; and finally preserving the computations $\{e\}\left(A \oplus W_{i} ; z\right)$ for $z<x$ by $A$-restraint alone. There are two difficulties with this. First, $C$ is a given set, so we do not have the freedom to literally put elements into $C$. However, $C$ is promptly simple and this fact can be exploited to force an element into $C$. The second problem is that computations using oracle $A \oplus W_{i}$ cannot be preserved by means of $A$-restraint alone. This means, as in the proof of Theorem 2.1, that we need to be prepared to try and fail infinitely many times to preserve some computations, translating our failures into evidence that $W_{i}$ is of promptly simple degree. As usual, such evidence consists of a total recursive function $p_{e, i}$ such that $W_{i}$ is of promptly simple degree via $p_{e, i}$.

The requirement $N_{e, i}$ is broken up into infinitely many requirements $N_{e, i, j^{\prime}}^{\prime}$, with $j$ varying over $N$. Requirement $N_{e, i}$ is equivalent to the conjunction of all the requirements $\left\{N_{e, i, j}^{\prime}: j \in \omega\right\}$, together with the condition that if $C=\{e\}\left(A \oplus W_{i}\right)$, then the function $p_{e, i}$ is total. 


$$
\begin{gathered}
N_{e, i, j}^{\prime}: C \neq\{e\}\left(A \oplus W_{i}\right) \\
\text { or } \\
(\exists y, s)\left[y \in W_{j, \text { at } s} \& W_{i, s}[y] \neq W_{i, p_{e, t}(s)}[y]\right] \\
\text { or } \\
W_{j} \text { is finite. }
\end{gathered}
$$

(In other words, if $C=\{e\}\left(A \oplus W_{i}\right)$ and $W_{j}$ is infinite, then there exists an element $y$ and a stage $s$ such that $y$ enters $W_{j}$ at stage $s$ and $W_{i}$ permits $y$ between stage $s$ and stage $p_{e, i}(s)$.)

We now turn to the basic strategy for the requirement $N_{e, i, j}^{\prime}$. This strategy involves building a auxiliary r.e. set $E_{e, i, j}$. By Remark 1.8, we may assume that we have an index $g(e, i, j)$ for $E_{e, i, j}$ available for use in the construction of $E_{e, i, j}$ and that numbers show up in $W_{g(e, i, j)}$ (strictly) later than they do in $E_{e, i, j}$.

The strategy for requirement $N_{e, i, j}^{\prime}$ is to be activated at a stage when a new, longer length of agreement is observed between $C$ and $\{e\}\left(A \oplus W_{i}\right)$-say $C(z)=$ $\{e\}\left(A \oplus W_{i} ; z\right)$ for all $z<x$. Then we begin waiting for some number

$$
y \geqslant u=\max \left\{\operatorname{use}\left(A \oplus W_{i} ; e, z\right): z<x\right\}
$$

to show up in $W_{j}$, say at stage $s$. Then at stage $s+1$, we perform the following steps. First we put $\bar{C}_{s}[x]$ into $E_{e, i, j}$. Next we find the earliest stage $t \geqslant s+1$ such that $W_{g(e, i, j), t}$ contains $E_{e, i, j, s+1}$. Then we see whether $C$ will promptly hit $W_{g(e, i, j)}$-i.e. whether $C_{s}[x] \neq C_{t+1}[x]$. If so, then, still at stage $s+1$, we impose $A$-restraint through $u=\max \left\{\operatorname{use}\left(A \oplus W_{i}: e, z\right): z<x\right\}$. In this case, we shall meet requirement $N_{e, i, j}^{\prime}$ either because by stage $t+1$ there will be a disagreement $C(z) \neq$ $\{e\}\left(A \oplus W_{i} ; z\right)$ for some $z<x$, and this disagreement will be indefinitely preserved because $C(z)=1$, or else because $W_{i}$ changes below $u$ at some stage $v+1 \geqslant s+1$, at which point we define $p_{e, i}(s)$ to be $v+1$, witnessing that $W_{i}$ has $p_{e, i}$-promptly permitted $y$. In the contrary case, i.e. where $C$ will not promptly hit $W_{g(e, i, j)}$, we have to deactivate the strategy and then start all over again when a new length of agreement longer than $x$ is recorded. Note that if $W_{j}$ is infinite and $C=$ $\{e\}\left(A \oplus W_{i}\right)$, then we will eventually activate the strategy at a stage $s+1$ where $C$ can be expected to promptly hit $W_{g(e, i, j)}$; for otherwise, $E_{e, i, j}=W_{g(e, i, j)}$ is made infinite but $C$ never promptly hits it.

It can be readily seen that the above strategy will meet requirement $N_{e, i, j}^{\prime}$. We denote by $r_{e, i, j}$ the restraint imposed on $A$ for the sake of requirement $N_{e, i, j}^{\prime}$.

As we put the requirements together, we run into conflicts which we will resolve by means of a priority ordering in which:

(1) $N_{e^{\prime}, i^{\prime}, j^{\prime}}^{\prime}$ is stronger than $N_{e, i, j}^{\prime}$ (denoted $\left.N_{e^{\prime}, i^{\prime}, j^{\prime}}^{\prime}\right\rangle N_{e, i, j}^{\prime}$ ) just if $\left\langle e^{\prime}, i^{\prime}, j^{\prime}\right\rangle<$ $\langle e, i, j\rangle$;

(2) $P_{n^{\prime}}>P_{n}$ just if $n^{\prime}<n$; and

(3) $N_{e, i, j}^{\prime}>P_{n}$ just if $\langle e, i, j\rangle \leqslant n$.

There will be only finitely many injuries to any requirement $N_{e, i, j}^{\prime}$. Requirements $P_{n}$ are never injured, of course. 
We say that requirement $P_{n}$ requires attention at Step 1 of stage $s+1$ if $P_{n}$ is not satisfied, and for some $y \in \omega$, all of the following were true at the end of stage $s$ :

(1) $y \geqslant \max \left\{r_{e, i, j}: N_{e, i, j}^{\prime}>P_{n}\right\}$;

(2) $y \geqslant 2 n$;

(3) $y \in W_{n \text {.at } s}$.

Requirement $N_{e, i, j}^{\prime}$ requires attention at Step 2 of stage $s+1$ if the restraint $r_{e, i, j}=-1$ and there exist $x, y \in \omega$ such that the following were true by the end of Step 1 of stage $s+1$ :

(1) $C(z)=\{e\}\left(A \oplus W_{i} ; z\right)$ for all $z<x$;

(2) $z \in\left(\overline{C \cup E_{e, i, j, s}}\right)$, for some $z<x$;

(3) $y \geqslant \max \left\{\operatorname{use}\left(A \oplus W_{i}\right.\right.$; e, $\left.\left.z\right): z<x\right\}$;

(4) $y \in W_{j \text {.ats }}$.

Here is the actual construction.

Construction. Stage $s=0$. (Do nothing.) For each $e, i, j \in \omega$, set the restraint $r_{e, i, j}$ to -1 .

Stage $s+1$.

Step 1. (Pay attention to at most one requirement $P_{n}$.) If some requirement $P_{n}$ requires attention, then choose the strongest requirement $P_{n}$ which requires attention and fix $y$ as small as possible. Put $y$ into $A$. Cancel all attacks and reset restraints to -1 for all requirements $N_{e, i, j}^{\prime}<P_{n}$.

Step 2. (Begin attacks for the requirements $N_{e, i, j}^{\prime}$.) For each requirement $N_{e, i, j}^{\prime}$ which requires attention, find the smallest possible $y$, and then the largest possible $x$. Put $\bar{C}_{s}[x]$ into $E_{e, i, j}$. Find the earliest stage $t \geqslant s+1$ such that $W_{g(e, i, j), t}$ contains $E_{e, i, j, s+1}$. Determine whether $C_{s}[x] \neq C_{t+1}[x]$. If so, appoint the tuple $\langle y, s, e, i, j\rangle$ as an attacker for requirement $N_{e, i, j}^{\prime}$ and set the restraint $r_{e, i, j}$ to

$$
\max \left\{\operatorname{use}\left(A \oplus W_{i}\right): z<x\right\} .
$$

Step 3. (End attacks for the requirements $N_{e, i, j}^{\prime}$.) Now for each requirement $N_{e, i . j}^{\prime}$ which has an uncancelled attacker $\left\langle y, s^{\prime}, e, i, j\right\rangle$, check whether $W_{i, s+1}[y] \neq W_{i, s}[y]$. If so, then end the attack by defining $p_{e, i}\left(s^{\prime}\right)$ to be $s+1$ and cancelling the attacker $\left\langle y, s^{\prime}, e, i, j\right\rangle$. To make the function $p_{e, i}$ total, also define $p_{e, i}$ on any arguments less than $s^{\prime}$ for which $p_{e, i}$ is not yet defined. (Do not reset the restraint $r_{e, i, j}$ to -1 ; the fact that $r_{e, i, j} \geqslant 0$ prevents requirement $N_{e, i, j}^{\prime}$ from requiring attention every again.)

END OF CONSTRUCTION. The proof is completed by means of a sequence of lemmas.

LEMMA 3.2. Every requirement $P_{n}$ and every requirement $N_{e, i, j}^{\prime}$ requires attention at most finitely many times. Every restraint $r_{e, i, j}$ has a final value to be denoted $\lim r_{e, i, j}$.

Proof. Fix a requirement $R$ and assume that $s_{0}$ is a stage so late that no requirement stronger than $R$ requires attention after stage $s_{0}$. We distinguish two cases:

Case $1 . R=P_{n}$.

If requirement $P_{n}$ ever requires attention at some stage $s+1>s_{0}$, then it will receive attention at that stage and never again require attention. 
Case 2. $R=N_{e, i, j}^{\prime}$.

Suppose that requirement $N_{e, i, j}^{\prime}$ requires attention infinitely often after stage $s_{0}$. If some attacker $\langle y, s, e, i, j\rangle$ with $s \geqslant s_{0}$ were eventually launched, then the restraint $r_{e, i, j}$ would assume some value $u \geqslant 0$ at stage $s+1$. Since $r_{e, i, j}$ would never later be injured, $\lim r_{e, i, j}=u$ the requirement $N_{e, i, j}^{\prime}$ would never require attention after stage $s+1$, contradiction. It follows that no attacker $\langle y, s, e, i, j\rangle$ with $s \geqslant s_{0}$ will ever be appointed. But then $E_{e, i, j}$ will end up infinite, and $C$ must promptly hit $W_{g(e, i, j)}=E_{e, i, j}$ infinitely many times-in particular, it must do so sometime after stage $s_{0}$. So some attacker will be launched after stage $s_{0}$. This contradiction means that requirement $N_{e, i, j}^{\prime}$ can only require attention finitely often and that the restraint $r_{e, i, j}$ has a final value.

LEMMA 3.3. Every requirement $P_{n}$ and every requirement $N_{e, i, j}^{\prime}$ is met.

Proof. Again fix a requirement $R$.

Case 1. $R=P_{n}$.

Let $s_{0}$ be a stage so late that no restraint $r_{e, i, j}$ which requirement $P_{n}$ must respect ever changes and no requirement $P_{n^{\prime}}>P_{n}$ requires attention after $s_{0}$. Then either no number $y$ with $y>\max \left\{r_{e, i, j}:\langle e, i, j\rangle \leqslant n\right\}$ and $y>2 n$ ever enters $W_{n}$ after stage $s_{0}$ so that $W_{n}$ is finite, or else requirement $P_{n}$ will require attention, receive attention, and become satisfied sometime after stage $s_{0}$.

Case 2. $R=N_{e, i, j}^{\prime}$ and $\lim r_{e, i, j} \geqslant 0$.

Let $s+1$ be the last stage during which requirement $N_{e, i, j}^{\prime}$ requires attention; let $x$ and $y$ be the numbers used in paying attention to $N_{e, i, j}^{\prime}$ at stage $s+1$. Then either requirement $N_{e, i, j}^{\prime}$ is met because there is a disagreement $C(z) \neq\{e\}\left(A \oplus W_{i} ; z\right)$ for some $z<x$ established at some point after stage $s+1$ and forever maintained afterward, or else there is a stage $v+1 \geqslant s+1$ such that $w_{i, v+1}[y] \neq W_{i, v}[y]$, so that requirement $N_{e, i, j}^{\prime}$ is met by defining $p_{e, i}(s)$ to be $v+1$.

Case 3. $R=N_{e, i, j}^{\prime}$ and $\lim r_{e, i, j}=-1$.

Fix a stage $s_{0}$ so late that the restraint $r_{e, i, j}=-1$ throughout all stages $s \geqslant s_{0}$. Then $C=\{e\}\left(A \oplus W_{i}\right)$ and $W_{j}$ infinite would imply that requirement $N_{e, i, j}^{\prime}$ would require attention infinitely often, contradicting Lemma 3.2. Thus either $C \neq$ $\{e\}\left(a \oplus W_{i}\right)$ or $W_{j}$ is finite and requirement $N_{e, i, j}^{\prime}$ is met trivially in this case.

LEMMA 3.4. Every requirement $N_{e, i}$ is met.

Proof. The only thing to check is that if $C=\{e\}\left(A \oplus W_{i}\right)$, then $p_{e, i}$ is total. This is evident from Step 3 of the construction.

This concludes the proof of the theorem.

\section{COROLlary 3.5. There are no minimal elements in $\underline{R} / \underline{M}$.}

Proof. Suppose that $\underline{c}_{\underline{M}}>\underline{0}_{\underline{M}}$. Apply Theorem 3.1 to $\underline{c}$, producing $\underline{a}_{\underline{M}}>\underline{0}_{M}$ such that $\underline{a}_{M} \neq \underline{c}_{M}$. Since $\underline{P}$ is a strong filter there exists an r.e. degree $\underline{b}$ such that $\underline{b}_{\underline{M}}>\underline{0}_{\underline{M}}, \underline{b}_{\underline{M}} \leqslant \underline{a}_{\underline{M}}$, and $\underline{b}_{\underline{M}} \leqslant \underline{c}_{\underline{M}}$. Now $\underline{b}_{\underline{M}}=\underline{c}_{\underline{M}}$ would imply that $\underline{c}_{\underline{M}} \leqslant \underline{a}_{\underline{M}}$ so $\underline{0}_{\underline{M}}<\underline{b}_{\underline{M}}<\underline{c}_{\underline{M}}$ and $\underline{c}_{\underline{M}}$ is not minimal in $\underline{R} / \underline{M}$. 
REMARK 3.6. There are no minimal pairs, triples, etc. in $\underline{R} / \underline{M}$.

Proof. Supose given $\underline{a}_{M}$ and $\underline{b}_{M}$ both $>\underline{0}_{M}$. By the strong filter property on $\underline{P}$ there exists a promptly simple r.e. degree $\underline{c}$ such that $\underline{c}_{M}>\underline{0}_{\underline{M}}, \underline{c}_{M} \leqslant \underline{a}_{\underline{M}}$ and $\underline{c}_{\underline{M}} \leqslant \underline{b}_{\underline{M}}$. Thus $\underline{a}_{\underline{M}}$ and $\underline{b}_{\underline{M}}$ do not form a minimal pair. The argument may be readily extended to rule out minimal triples, etc. We leave this to the reader.

4. The splitting theorem for $\underline{R} / \underline{M}$. This section presents the proof of the $\underline{R} / \underline{M}$-counterpart to Sacks' splitting theorem. We also give two corollaries.

TheOREM 4.1 (The SPlitTing THeOREM). Given r.e. sets $B$ and $C$, with $C$ promptly simple, then $B$ can be expressed as the disjoint union of two r.e. sets $A_{0}$ and $A_{1}$ where

$$
\left(\operatorname{deg} A_{0}\right)_{\underline{M}} \ngtr(\operatorname{deg} C)_{\underline{M}} \text { and }\left(\operatorname{deg} A_{1}\right)_{\underline{M}} \ngtr(\operatorname{deg} C)_{\underline{M}} .
$$

Proof. Let $\left\{b_{s}: s \in \omega\right\}$ be a one-to-one recursive enumeration of the r.e. set $B$. Assume given a recursive enumeration of the r.e. set $C$ for which $p(s)=s+1$ is a prompt simplicity function. We shall build r.e. sets $A_{0}$ and $A_{1}$ to meet, for all $m \in\{0,1\}$ and all $e, i, s \in \omega$, the following requirements:

$$
\begin{gathered}
P_{s}: b_{s} \in A_{0} \text { or } b_{s} \in A_{1} \text { (but not both). } \\
N_{m, e, i}: C \neq\{e\}\left(A_{m} \oplus W_{i}\right) \\
\text { or }
\end{gathered}
$$

$W_{i}$ is of promptly simple degree.

Evidently, if we only had to meet requirements $P_{s}$, we could, say, always choose to put $b_{s}$ into $A_{0}$. It is the interaction with the requirements $N_{m, e, i}$ which forces us to employ both sets $A_{0}$ and $A_{1}$ in meeting requirement $P_{s}$.

Fix $m \in\{0,1\}$. Then the requirements $\left\{N_{m, e, i}: e, i \in \omega\right\}$ are entirely analogous to the requirements $\left\{N_{e, i}: e, i \in \omega\right\}$ in the proof of Theorem 3.1, and we shall handle them with precisely the same techniques. Thus we divide each requirement $N_{m, e, i}$ up into requirements $N_{m, e, i, j}^{\prime}$, where $j$ varies over $N$.

$$
\begin{gathered}
N_{m, e, i, j}^{\prime}: C \neq\{e\}\left(A_{m} \oplus W_{i}\right) \\
\text { or } \\
(\exists y, s)\left[y \in W_{j, \text { at } s} \& W_{i, s}[y] \neq W_{i, p_{m, e, u}(s)}[y]\right] \\
\text { or } \\
W_{j} \text { is finite. }
\end{gathered}
$$

(In other words, if $C=\{e\}\left(A_{m} \oplus W_{i}\right)$ and $W_{j}$ is infinite, then there exist an element $y$ and a stage $s$, such that $y$ enters $W_{j}$ at stage $s$ and $W_{i}$ permits $y$ between stage $s$ and stage $p_{m, e, i}(s) ; p_{m, e, i}$ is the total recursive function which is intended to witness that $W_{i}$ is promptly simple in degree, in case $C=\{e\}\left(A_{m} \oplus W_{i}\right)$.) The strategy for meeting requirement $N_{m, e, i, j}^{\prime}$ is just like that for meeting requirement $N_{e, i, j}^{\prime}$ from Theorem 3.1. In particular, we shall build auxiliary r.e. sets $E_{m, e, i, j}=W_{g(m, e, i, j)}$ in order to exploit the prompt simplicity of $C$, and $r_{m, e, i, j}$ will denote the restraint imposed on $A_{m}$ for the sake of requirement $N_{m, e, i, j}^{\prime}$.

As we put the requirements together, we run into conflicts. We establish the priority ranking on the requirements $N_{m, e, i . j}^{\prime}$ in which $N_{m, e, i, j}^{\prime}$ is stronger than 
$N_{m^{\prime}, e^{\prime}, i^{\prime}, j^{\prime}}^{\prime}\left(\operatorname{denoted} N_{m, e, i, j}^{\prime}>N_{m^{\prime}, e^{\prime}, i^{\prime}, j^{\prime}}^{\prime}\right)$ just if $\langle m, e, i, j\rangle\left\langle\left\langle m^{\prime}, e^{\prime}, i^{\prime}, j^{\prime}\right\rangle\right.$. Instead of giving the requirements $P_{s}$ any particular static priority rankings, however, we shall use a dynamic ranking as follows. At stage $s+1$, when deciding whether $b_{s}$ should go into $A_{0}$ or $A_{1}$, we will look to see which requirement $N_{m, e, i, j}^{\prime}$ is the strongest among all those (if any) such that $b_{s}<r_{m, e, i, j}$. Then we put $b_{s}$ into $A_{1-m}$, avoiding injury to requirement $N_{m, e, i, j}^{\prime}$. Note that this same dynamic ranking of positive requirements was used in Sacks' original splitting theorem. (See Soare [1980] for a clear account of the proof of Sacks' splitting theorem.) There will be only finitely many injuries to each $N_{m, e, i, j}^{\prime}$. Requirements $P_{s}$ are never injured, of course.

The conditions under which requirement $N_{m, e, i, j}^{\prime}$ requires attention at Step 2 of stage $s+1$ are the same as those for requirement $N_{e, i, j}^{\prime}$ of Theorem 3.1. At Step 1 of stage $s+1$, we shall give attention to (and satisfy) requirement $P_{s}$.

Here is the actual construction.

Construction. Stage $s=0$. (Do nothing.) For each $m \in\{0,1\}$ and each $e, i, j$ $\in \omega$, set the restraint $r_{m, e, i, j}$ to -1 .

Stage $s+1$.

Step 1. (Satisfy $P_{s}$.) If there are any restraints $r_{m, e, i, j}>b_{s}$, then choose the strongest requirement $N_{m, e, i, j}^{\prime}$ whose restraint $r_{m, e, i, j}>b_{s}$, and put $b_{s}$ into $A_{1-m}$. (Otherwise, put $b_{s}$ into $A_{0}$.) Cancel attackers and reset restraints to -1 for any requirements $N_{1-m, e^{\prime}, i^{\prime}, j^{\prime}}$, which have just been injured by the entrance of $b_{s}$ into $A_{1-m}$.

Steps 2 and 3. (Open and close attacks for the requirements $N_{m, e, i}^{\prime}$ ) These steps are identical to the corresponding steps of stage $s+1$ in the construction from the proof of Theorem 3.1.

END OF Construction. The proof is completed by means of a sequence of lemmas.

LEMMA 4.2. Every requirement $P_{s}$ and every requirement $N_{m, e, i, j}^{\prime}$ requires attention at most finitely often. Every restraint $r_{m, e, i, j}$ has a final value to be denoted $\lim r_{m, e, i, j}$.

Proof. Clearly, each $P_{s}$ requires attention precisely once, namely at stage $s+1$. So fix a requirement $N_{m, e, i, j}^{\prime}$ and assume inductively that $s_{0}$ is a stage so late that for all stages $s \geqslant s_{0}, b_{s} \geqslant r$, where $r$ is the maximum of all of the values ever assumed by all the restraints $r_{m^{\prime}, e^{\prime}, i^{\prime}, j^{\prime}}$, of requirements stronger than $N_{m, e, i, j}^{\prime}$. Then $N_{m, e, i, j}^{\prime}$ can never be injured after stage $s_{0}$. The rest of the proof of this lemma now proceeds as in Case 2 of the proof of Lemma 3.2 from Theorem 3.1.

LEMMA 4.3. Every requirement $P_{s}$ and every requirement ${N^{\prime}, e, i, j}^{\prime}$ is met.

Proof. This is obvious for requirements $P_{s}$. The proof for requirements ${N^{\prime}}_{m, e, i, j}$ proceeds just as in Cases 2 and 3 of Lemma 3.3 from Theorem 3.1.

LEMMA 4.4. Every requirement $N_{m, e, i}$ is met.

PRoof. The proof is the same as the proof of Lemma 3.4 from Theorem 3.1.

This concludes the proof of the theorem.

COROLLARY 4.5. Any $\underline{b}_{\underline{M}}>\underline{0}_{\underline{M}}$ in $\underline{R} / \underline{M}$ can be expressed as the join of incomparable elements $\left(\underline{a}_{0}\right)_{\underline{M}}$ and $\left(\underline{a}_{1}\right)_{\underline{M}}$ of $\underline{R} / \underline{M}$. 
Proof. Pick a promptly simple r.e. set $B \in \underline{b}$ and apply Theorem 4.1 with $C=B$. Let $\underline{a}_{m}=\operatorname{deg} A_{m}$ for $m \in\{0,1\}$. We claim that $\underline{a}_{0}$ and $\underline{a}_{1}$ will do.

Since $B, A_{0}$, and $A_{1}$ are r.e. and $B$ is the disjoint union of $A_{0}$ and $A_{1}$, we have that $B \equiv{ }_{T} A_{0} \oplus A_{1}$. Thus $\underline{b}=\underline{a}_{0} \vee \underline{a}_{1}$ and so certainly $\underline{b}_{M}=\left(\underline{a}_{0} \vee \underline{a}_{1}\right)_{M}=$ $\left(\underline{a}_{0}\right)_{\underline{M}} \vee\left(\underline{a}_{1}\right)_{\underline{M}}$. Now if, say $\left(\underline{a}_{0}\right)_{\underline{M}} \leqslant\left(\underline{a}_{1}\right)_{\underline{M}}$, then $\underline{b}_{M}=\left(\underline{a}_{0}\right)_{\underline{M}} \vee\left(\underline{a}_{1}\right)_{\underline{M}}=\left(\overline{a_{1}}\right)_{\underline{M}}$, contradicting $\left(\underline{a}_{1}\right)_{\underline{M}} \ngtr \underline{b}_{\underline{M}}$.

COROLlaRY 4.6. For any $\underline{c}_{\underline{M}}$ in $\underline{R} / \underline{M}$ such that $\underline{0}_{\underline{M}}<\underline{c}_{\underline{M}}<\underline{0}_{\underline{M}}^{\prime}$, there is an element $\underline{a}_{\underline{M}}$ of $\underline{R} / \underline{M}$ which is incomparable with $\underline{c}_{\underline{M}}$.

Proof. Pick a promptly simple r.e. set $C \in \underline{c}$. Apply Theorem 4.1 to $B=\varnothing^{\prime}$ and $C$. Let $\underline{a}_{m}=\operatorname{deg} A_{m}$ for $m \in\{0,1\}$. We claim that either $\underline{a}_{M}=\left(\underline{a}_{0}\right)_{\underline{M}}$ or $\underline{a}_{M}=\left(\underline{a}_{1}\right)_{\underline{M}}$ will do.

For suppose that both $\left(\underline{a}_{0}\right)_{\underline{M}}$ and $\left(\underline{a}_{1}\right)_{\underline{M}}$ were comparable with $\underline{c}_{\underline{M}}$. Since $\left(\underline{a}_{m}\right)_{\underline{M}} \neq$ $\underline{c}_{\underline{M}}$, for $m \in\{0,1\}$, it follows that $\left(\underline{a}_{m}\right)_{\underline{M}} \leqslant \underline{c}_{\underline{M}}$ for $m \in\{\overline{0}, 1\}$. But $\underline{b}_{\underline{M}}=$ $\left(\underline{a}_{0} \vee \underline{a}_{1}\right)_{\underline{M}}$ just as in the proof of the previous corollary, so $\underline{0}^{\prime} \underline{M}=\underline{b}_{M}=$ $\left(\underline{a}_{0}\right)_{\underline{M}} \vee\left(\underline{a}_{1}\right)_{\underline{M}} \leqslant \underline{c}_{\underline{M}}$, a contradiction.

\section{REFERENCES}

K. Ambos-Spies, On the structure of the recursively enumerable degrees, Ph. D. thesis, University of Munich, 1980.

K. Ambos-Spies, C. Jockusch, R. Shore and R. Soare, An algebraic decomposition of the recursicely. enumerable degrees and classes equal to the promptly simple degrees (to appear).

R. Friedberg, Two recursively enumerable sets of incomparable degrees of unsolvability. Proc. Nat. Acad. Sci. U.S.A. 43 (1957).

A. Lachlan, Lower bounds for pairs of r.e. degrees, Proc. London Math. Soc. 16 (1966).

W. Maass, Recursicely enumerable generic sets, J. Symbolic Logic (to appear).

W. Maass, R. Shore and M. Stob, Splitting properties and jump classes, Israel J. Math. 39 (1981).

A. A. Muchnik, On the unsolvability of the problem of reducibility in the theory of algorithms. Dokl. Akad. Nauk SSSR 108 (1956). (Russian)

G. Sacks. On the degrees less than 0', Ann. of Math. (2) 77 (1963). , The recursively enumerable degrees are dense, Ann. of Math. (2) 80 (1964).

Degrees of unsolvability, rev. ed. Ann. of Math. Studies, no. 55. Princeton Univ. Press.

Princeton, N. J., 1966.

J. Shoenfield, Application of model theory to degrees of unsolvability. Sympos. Theory of Models, North-Holland, Amsterdam, 1965.

R. Soare, The infinite injury priority method, J. Symbolic Logic 41 (1976). , Recursively enumerable sets and degrees, Bull. Amer. Math. Soc. 84 (1978).

Fundamental methods for constructing recursively enumerable degrees, Recursion Theory, Its Generalizations and Applications (Eds., F. Drake and S. Wainer), Cambridge Univ. Press. New York. 1980.

R. Soare, Computational complexity of recursively enumerable sets (to appear).

C. E. M. Yates, A minimal pair of r.e. degrees, J. Symbolic Logic 31 (1966).

Department of Mathematics, Massachusetts institute of Technology, Cambridge, MasSACHUSETTS 02139

Current address: Department of Mathematics, Tufts University, Medford, Massachusetts 02155 